\title{
Numerical investigation of a Stokes model for flow down a fiber
}

\author{
Dennis S. Fillebrown \\ Department of Mathematics, Bucknell University, Lewisburg, Pennsylvania 17837, USA \\ dsf007@bucknell.edu \\ Advisor: Linda B. Smolka \\ Department of Mathematics, Bucknell University, Lewisburg, Pennsylvania 17837, USA \\ lsmolka@bucknell.edu
}

We numerically solve a fourth order nonlinear partial differential equation derived by Craster and Matar [Craster and Matar, J. Fluid Mech. 553, 85 (2006)] that models a viscous fluid flowing down the outside of a vertical fiber in order to investigate the initial formation of perturbations along the fluid free surface. We compare numerical results of their model to existing experimental data [Smolka et al., Phys. Rev. E 77, 036301 (2008)]. In the simulations, perturbations consistently coalesced with neighboring perturbations during their initial formation, whereas in the experiments no coalescence was observed during this time period. We find that the amplitude growth follows two distinct exponential functions (referred to as phases I and II) in the initial formation of perturbations; in the experiments the data follows only one exponential function. The switch in growth rates from phase I to II is influenced by the coalescence of neighboring perturbations. The wavelength varies throughout the time that a perturbation forms so that the flow is unstable to a range of wavenumber. We compare the growth rates of several perturbations during phase II from the simulations to experimental data. Of the six data sets, the range of growth rate and wavenumber were in excellent agreement in two of the data sets and in fair to good agreement with two other data sets. For the last two data sets, there was no overlap in growth rate and little overlap in wavenumber. We find that linear stability results developed from Craster and Matar's Stokes flow model are in excellent qualitative agreement with the growth of the perturbations measured during phase II in simulations for all six data sets. Finally, we find that data from simulations consistently overpredict the final perturbation amplitude measured in experiments.

\section{INTRODUCTION}

We investigate a viscous fluid flowing down the outside of a thin vertical fiber, as shown in Fig. 1. Numerous analytical and experimental studies [1-6] have been conducted to study the motion and stability of this flow. Figure 1 shows that somewhere down the length of the fiber the free surface becomes unstable to interfacial or "wave-like" perturbations [1-4]. Depending on the fiber radius and flow rate at the fluid source, the behavior of the perturbations including their size, speed, and interactions with one another can be quite different [1-4]. Understanding the dynamics of these perturbations is important to controlling such flows in manufacturing and industrial processes, e.g., wire coating [7-10]. In this paper, we focus on the initial formation of the perturbations.

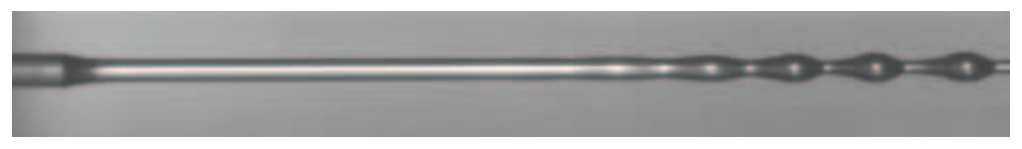

FIG. 1: A viscous flow down the outside of a thin vertical fiber; the surrounding fluid is air. The figure has been rotated by $90^{\circ}$ with gravity acting toward the right. Perturbations develop along the free surface some distance from the fluid source. Once formed, these perturbations continue to travel down the fiber. Image length is $9.7 \mathrm{~cm}$.

In their experimental study with castor oil, Kliakhandler, Davis and Bankoff (KDB) [1] focused on the dynamics of the perturbations $2 \mathrm{~m}$ down the fiber from the fluid source. They observed three distinct types of behavior depending on the flow rate. At high flow rates, large droplets with nearly uniform film thickness between the droplets were observed. At intermediate flow rates, smaller, evenly spaced droplets formed that moved with constant velocity. At low flow rates, the flow dripped with large droplets forming periodically. The long time between droplets allowed the film between the perturbations to thin and become unstable to even smaller interfacial perturbations. At high flow rates the perturbations were observed to coalesce with one another, while at intermediate flow rates no coalescence was observed. KDB derived a Stokes flow model for the evolution of the free surface, thus neglecting inertial effects, that is asymptotically valid for thin film thicknesses [1]. They found good qualitative agreement between numerical results of the model and experiments at intermediate and low flow rates, however, their model could not reproduce the behavior at high flow rates [1]. 
In their experimental investigation with silicone oil, Craster and Matar (CM) [2] did not observe the distinct behavior corresponding to different flow rates that KDB observed, but rather a gradual shift between the behaviors. In particular, at the intermediate flow rate they observed evenly spaced droplets near the fluid source, but further down the fiber the regularly spaced pattern of perturbations disassembled itself. Craster and Matar selected a different scaling than KDB to derive an evolution equation for the free surface. Their Stokes flow model is valid when the Reynolds number is $O(1)$ and the Bond number is much smaller than 1 [2]. Using traveling wave solutions, their model quantitatively predicted the perturbation speed and height measured by KDB at the high flow rates. Their model also qualitatively captured the dynamics at the low flow rates. The steady pattern of perturbation spacing found at intermediate flow rates by KDB could not, however, be matched with traveling wave solutions.

In contrast to previous experimental studies [1, 2], Smolka, North, and Guerra (SNG) focused on the initial formation of perturbations near the fluid source (orifice) for both Stokes and moderate flow rates [3]. To illustrate the dynamics they observed, a sequence of images from their experiments with castor oil and data collected from these images are shown in Figs. 2 and 3. Figure 2(a) shows that the free surface is spatially uniform until about $5.5 \mathrm{~cm}$ down the fiber where perturbations initially appear. The sequence of images in Fig. 2 tracks the position of a perturbation as it grows (indicated by the ovaled region). SNG found that initially the perturbation's amplitude grows exponentially then slows over time until the amplitude saturates in size (see Fig. 3(a)); the data shown in Fig. 3 corresponds to the perturbation tracked in Fig. 2. The perturbation wavelength (measured between this perturbation and the one behind it) decreases during the time interval that the amplitude grows exponentially and then attains a constant value as the amplitude growth slows down, as shown in Fig. 3(b). In all of their experiments with castor oil, SNG found excellent agreement between the linear stability results obtained from Craster and Matar's Stokes flow model [2] and their experimental data during the exponential stage of amplitude growth [3].
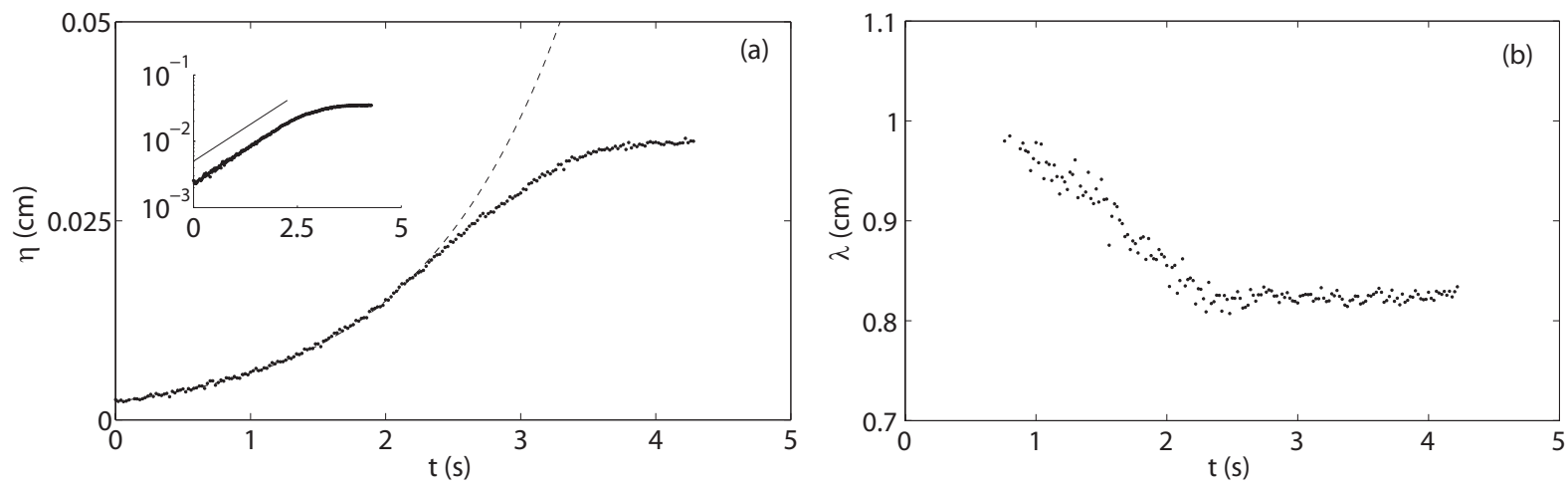

FIG. 3: (a) Amplitude and (b) wavelength as a function of time for the perturbation tracked in Fig. 2 (data set taken from the experimental study of SNG [3]). The initial amplitude growth is exponential, as shown in the inset, and then slows until the amplitude saturates in size. The time interval of exponential growth occurs between Fig. 2(a)-(g); a best fit of the data is given by $\eta(t)=0.0023 e^{0.935 t}$ (dashed line). The wavelength decreases during the period of exponential growth and then attains a constant value as the amplitude saturates in size.

Our focus will be on the dynamics of the perturbations near the orifice. Previous investigations of Craster and Matar's model have focused on the later time dynamics of the perturbations [2]. Here, we will use the CM model [2] to investigate the early time dynamics when the perturbations initially form. To test the validity of the CM model, we compare results from numerical simulations of their model to the experimental data collected by SNG [3]. 
The rest of the paper is organized as follows. In Section II, we describe the physical parameters of the flow and Craster and Matar's evolution equation for the fluid free surface. In Section III, we describe the free boundary problem that includes Craster and Matar's evolution equation with boundary and initial conditions, the numerical techniques used to solve the free boundary problem and values of the parameters used in the numerical simulations. In Section IV, we present numerical results and provide comparisons with experimental data collected by SNG [3]. In Section V, we summarize our findings.

\section{PHYSICAL PARAMETERS AND STOKES FLOW MODEL}

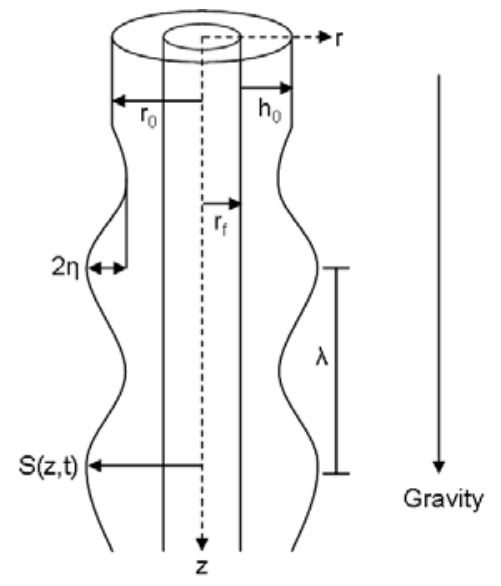

FIG. 4: Schematic of a viscous fluid flowing down the outside of a vertical fiber of radius $r_{f}$. The radial distance from the center of the fiber to the fluid free surface is $r=S(z, t)$. Near the fluid source, the flow is unperturbed with constant fluid thickness $r=h_{0}$ and radial distance to the free surface $r=r_{0}$. The perturbation amplitude is $\eta$ and the wavelength is $\lambda$.
We consider the flow of a viscous fluid down a fiber of radius $r_{f}$. The relevant properties of the fluid are the dynamic viscosity, $\mu$, the density, $\rho$, and the surface tension, $\gamma$. The free surface of the flow measured from the fiber axis is denoted by $r=S(z, t)$, the uniform film thickness near the orifice is denoted by $r=h_{0}$, and the uniform radial distance to the free surface near the orifice is denoted by $r=r_{0}$ (see Fig. 4). The perturbations are characterized by their amplitude $\eta$ and wavelength $\lambda$. The surrounding fluid is assumed to be an inviscid gas whose influence on the viscous flow is ignored [2].

The evolution equation describing the fluid free surface for this flow derived by Craster and Matar [2] is

$$
\begin{aligned}
& 8 \partial_{\hat{t}}\left(\hat{S}^{2}\right)+\partial_{\hat{z}}\left(\left[\partial_{\hat{z}}\left[\frac{1}{\hat{S}}-\epsilon^{2} \partial_{\hat{z} \hat{z}} \hat{S}\right]-1\right] \times\right. \\
& \left.\left[\alpha^{4}-4 \alpha^{2} \hat{S}^{2}+3 \hat{S}^{4}-4 \hat{S}^{4} \ln \left(\frac{\hat{S}}{\alpha}\right)\right]\right)=0,
\end{aligned}
$$

where $\alpha=r_{f} / r_{0}$ and the Bond number, $\epsilon=\left(\rho g r_{0}^{2}\right) / \gamma$, is a measure of the ratio of gravitational forces to surface tension forces. The dimensionless variables $\hat{S}, \hat{z}, \hat{t}$ are defined by

$$
\hat{S}=\frac{S}{r_{0}}, \quad \hat{z}=\frac{z}{\mathcal{L}}, \quad \hat{t}=\frac{\mathcal{V} t}{\mathcal{L}},
$$

where the characteristic lengthscale (the capillary length) and velocity scale are given by

$$
\mathcal{L}=\frac{\gamma}{\rho g r_{0}}, \quad \mathcal{V}=\frac{\rho g r_{0}^{2}}{\mu} .
$$

The model, which is a fourth order nonlinear partial differential equation (PDE), is valid under the conditions that the Reynolds number $(\operatorname{Re}=\rho \mathcal{V} \mathcal{L} / \mu)$ is $O(1)$ and $\epsilon \ll 1$. Since $\epsilon=r_{0} / \mathcal{L}$, the radial thickness of the unperturbed film, $r_{0}$, must be small relative to the capillary length, $\mathcal{L}[2]$. We numerically solve (1a) to examine the early time dynamics of the perturbations that develop along the free surface.

A solution of (1a) is $\hat{S}(\hat{z}, \hat{t})=1$ which represents a uniform unperturbed film flowing down the length of the fiber. Craster and Matar [2] conducted a linear stability analysis of (1a) by perturbing about this base flow

$$
\hat{S}(\hat{z}, \hat{t})=1+\hat{S}_{1} e^{(i \hat{m} \hat{z}+\hat{\sigma} \hat{t})},
$$

where $\hat{m}$ is the real dimensionless wavenumber and $\hat{\sigma}$ is the complex dimensionless growth rate. Substituting (2a) into (1a) and linearizing about the base flow yields the dispersion relation for the growth rate

$$
\hat{\sigma}=\frac{\hat{m}^{2}}{16}\left(\epsilon^{2} \hat{m}^{2}-1\right)\left[\alpha^{4}-4 \alpha^{2}+3+4 \ln (\alpha)\right]-\frac{i \hat{m}}{2}\left[\alpha^{2}-1-2 \ln (\alpha)\right] .
$$

This stability result developed by Craster and Matar is a temporal analysis (since $\hat{m}$ is real and $\hat{\sigma}$ is complex) and thus models the case in which interfacial perturbations grow in amplitude everywhere along the free surface [11]. In the experiments, perturbations do not grow everywhere along the free surface [3]. Instead, they initially form at one 


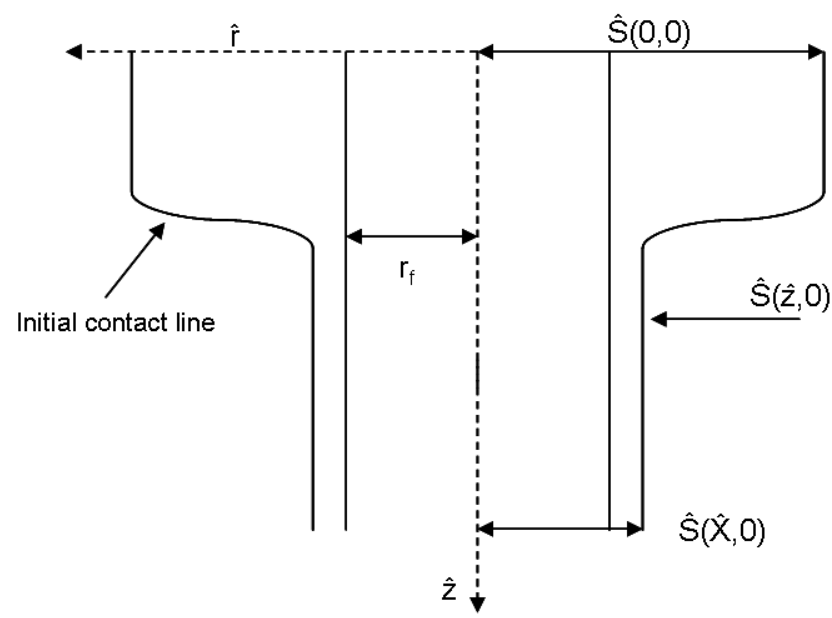

FIG. 5: Cross-section of the initial fluid profile, $\hat{S}(\hat{z}, 0)$, used in the numerical simulations (not drawn to scale). The free surface is uniform near the orifice $(\hat{z}=0)$ and transitions to a thin pre-wetted layer. The transition region represents the leading edge, or contact line, of the flow.

location along the fiber and then grow in amplitude as they travel down the fiber; this is a convective or spatial instability. Nevertheless, the real part of $\hat{\sigma}$ predicts the initial exponential growth of a perturbation, which SNG used to compare to their experimental data [3]. In a similar fashion, we will compare data from our numerical simulations of (1a) to the real part of $\hat{\sigma}$.

Before presenting results of our numerical simulations of Craster and Matar's model, we first describe details of the numerical modeling.

\section{DETAILS OF NUMERICAL MODELING}

To solve the fourth order nonlinear PDE (1a) numerically, we approximate the PDE using finite differences. Here we describe the boundary conditions, the initial condition, the discretization scheme for the fourth order nonlinear PDE (1a), the numerical techniques that we use to solve the resulting discretized nonlinear system of equations and the values of the parameters used in the numerical model.

In their simulations of (1a), Craster and Matar used periodic boundary conditions and focused on the long-time behavior of the perturbations well after their initial formation [2]. Here we use boundary conditions that partially represent the experimental conditions [3] and instead focus on the initial formation of the perturbations. Since (1a) is fourth order, we must provide four boundary conditions.

In our numerical study, we solve (1a) on $\hat{z} \in[0, \hat{X}]$, where $\hat{X}=X / \mathcal{L}$ represents the nondimensional fiber length. The boundary conditions at the top of the fiber, $\hat{z}=0$, are

$$
\hat{S}(0, \hat{t})=1 \quad \text { and } \quad \partial_{\hat{z}} \hat{S}(0, \hat{t})=0, \quad \forall \hat{t},
$$

corresponding to a flat film of prescribed thickness at the orifice. These boundary conditions are chosen to correspond to the experimental conditions [3]. In the experiment, the boundary conditions at the bottom of the fiber change in time as perturbations fall off the bottom of the fiber into a collection tank [3]. Since these conditions are difficult to model, we instead investigate the flow near the orifice while the leading edge of the fluid (called the contact line) travels down the fiber. The boundary conditions at the bottom of the fiber, $\hat{z}=\hat{X}$, that we use are

$$
\hat{S}(\hat{X}, \hat{t})=\alpha+0.1 \frac{h_{0}}{r_{0}} \quad \text { and } \quad \partial_{\hat{z}} \hat{S}(\hat{X}, \hat{t})=0, \quad \forall \hat{t},
$$

corresponding to the fiber having a flat, pre-wetted thin layer of fluid ahead of the contact line. Introducing a prewetted layer ahead of the contact line avoids the singularity that would otherwise form in the simulations at the fluid/air/solid interface (in the absence of a pre-wetted layer) due to the no-slip boundary condition [12]. 
The initial condition for the free surface, given by

$$
\hat{S}(\hat{z}, 0)=\frac{1}{2}\left[\left(1+\alpha+0.1 \frac{h_{0}}{r_{0}}\right)-\left(1-\alpha-0.1 \frac{h_{0}}{r_{0}}\right) \tanh (\hat{z}-0.1 \hat{X})\right] \quad \text { for } \quad 0<\hat{z}<\hat{X},
$$

represents a spatially uniform unperturbed free surface near the orifice that smoothly transitions to a spatially uniform thin pre-wetted layer; the transition region represents the leading edge, or contact line, of the fluid (see Fig. 5). The initial condition (3c) satisfies the boundary conditions (3a) and (3b). In the rest of the paper, all quantities will be presented in non-dimensional units unless otherwise stated, so for convenience we drop the hat notation.

To solve (1a) numerically, we discretize space and time. In space, we use a uniform spatial grid by dividing the length of the fiber into $\mathrm{N}$ equal subintervals of length $d z=X / N$ and denote the axial grid points by $z_{i}$ for $1 \leq i \leq N+1$, so that $z_{1}=0, z_{2}=d z, z_{3}=2 d z, \cdots, z_{N+1}=N d z=X$. In time, we specify the time step length to be $d t$ and denote a given time step by $t_{j}=j d t$, where $j$ is a nonnegative integer. At axial grid point $z_{i}$ and time step $t_{j}$, we denote the free surface by $S_{i, j}=S\left(z_{i}, t_{j}\right)$.

We approximate (1a) with finite differences using the fully implicit backward Euler method for the time derivative and centered differencing for the spatial derivatives. This discretization scheme is formally first order accurate in time and second order accurate in space. Since the highest spatial derivative in (1a) is fourth order, this discretization scheme requires the values $S_{i+2, j}, S_{i+1, j}, S_{i, j}, S_{i-1, j}, S_{i-2, j}$ and $S_{i, j-1}$ to compute the solution at $\left(z_{i}, t_{j}\right)$, that is, $S_{i, j}$ (a stencil is shown in Fig. 6).

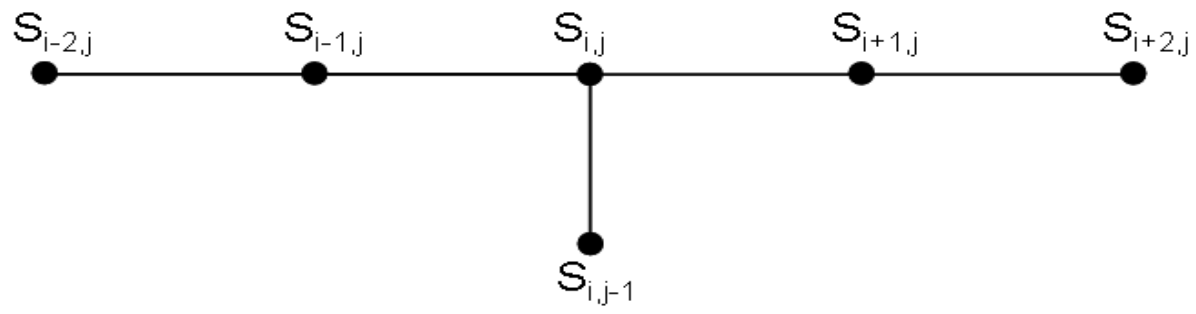

FIG. 6: The stencil of points needed to compute $S_{i, j}$.

The discretization of (1a) to compute $S_{i, j}$ is

$$
8\left(\frac{\left(S_{i, j}\right)^{2}-\left(S_{i, j-1}\right)^{2}}{d t}\right)+\left(\frac{G_{i+\frac{1}{2}, j}-G_{i-\frac{1}{2}, j}}{d z}\right)=0,
$$

where

$$
\begin{aligned}
G_{i+\frac{1}{2}, j} & =A_{i+\frac{1}{2}, j} \times\left[\alpha^{4}-4 \alpha^{2}\left(S_{i+\frac{1}{2}, j}\right)^{2}+3\left(S_{i+\frac{1}{2}, j}\right)^{4}-4\left(S_{i+\frac{1}{2}, j}\right)^{4} \ln \left(\frac{1}{\alpha} S_{i+\frac{1}{2}, j}\right)\right], \\
G_{i-\frac{1}{2}, j} & =A_{i-\frac{1}{2}, j} \times\left[\alpha^{4}-4 \alpha^{2}\left(S_{i-\frac{1}{2}, j}\right)^{2}+3\left(S_{i-\frac{1}{2}, j}\right)^{4}-4\left(S_{i-\frac{1}{2}, j}\right)^{4} \ln \left(\frac{1}{\alpha} S_{i-\frac{1}{2}, j}\right)\right], \\
A_{i+\frac{1}{2}, j} & =\frac{1}{d z}\left(\frac{1}{S_{i+1, j}}-\frac{1}{S_{i, j}}\right)-\epsilon^{2}\left(\frac{S_{i+2, j}-3 S_{i+1, j}+3 S_{i, j}-S_{i-1, j}}{d z^{3}}\right)-1, \\
A_{i-\frac{1}{2}, j} & =\frac{1}{d z}\left(\frac{1}{S_{i, j}}-\frac{1}{S_{i-1, j}}\right)-\epsilon^{2}\left(\frac{S_{i+1, j}-3 S_{i, j}+3 S_{i-1, j}-S_{i-2, j}}{d z^{3}}\right)-1, \\
S_{i+\frac{1}{2}, j} & =\frac{S_{i, j}+S_{i+1, j}}{2} \\
S_{i-\frac{1}{2}, j} & =\frac{S_{i, j}+S_{i-1, j}}{2}
\end{aligned}
$$

for $2 \leq i \leq N$.

In discretized form, the boundary conditions at $z_{1}=0(3 \mathrm{a})$ are

$$
\begin{aligned}
& S\left(0, t_{j}\right)=1, \quad \forall t_{j} \quad \Longrightarrow \quad S_{1, j}=1, \quad j \geq 0, \\
& \partial_{z} S\left(0, t_{j}\right)=0, \quad \forall t_{j} \quad \Longrightarrow \quad S_{0, j}=S_{2, j}, \quad j \geq 0,
\end{aligned}
$$


and the boundary conditions at $z_{N+1}=X(3 \mathrm{~b})$ are

$$
\begin{gathered}
S\left(X, t_{j}\right)=\alpha+0.1 \frac{h_{0}}{r_{0}}, \quad \forall t_{j} \quad \Longrightarrow \quad S_{N+1, j}=\alpha+0.1 \frac{h_{0}}{r_{0}}, \quad j \geq 0 \\
\partial_{z} S\left(X, t_{j}\right)=0, \quad \forall t_{j} \quad \Longrightarrow \quad S_{N+2, j}=S_{N, j}, \quad j \geq 0 .
\end{gathered}
$$

Note that the axial points $z_{0}=-d z$ (in $\left.(5 \mathrm{~b})\right)$ and $z_{N+2}=(N+1) d z($ in $(5 \mathrm{~d}))$ extend beyond the physical fiber and are needed to solve (4) for $S_{2, j}$ and $S_{N, j}$, respectively. The discretized form of the initial condition is

$$
S_{i, 0}=\frac{1}{2}\left[\left(1+\alpha+0.1 \frac{h_{0}}{r_{0}}\right)-\left(1-\alpha-0.1 \frac{h_{0}}{r_{0}}\right) \tanh \left(z_{i}-0.1 z_{N+1}\right)\right] \quad \text { for } \quad 0<i<N+1 .
$$

The discretized free boundary problem, (4) and (5), forms a system of $N+1$ nonlinear equations in $N+1$ unknowns, which can be expressed in vector form as

$$
\vec{F}\left(\vec{S}_{j}\right)=\overrightarrow{0}
$$

where $\vec{F}=\left[F_{1}, F_{2}, \cdots, F_{N+1}\right]^{T}$ and the unknowns are $\vec{S}_{j}=\left[S_{1, j}, S_{2, j}, S_{3, j}, \cdots, S_{N+1, j}\right]^{T}$. To solve (6) at each time step $t_{j}$ we use Newton's Iterative Method [13]. For the first iteration $(k=0)$, we make an initial guess for the solution $\vec{S}_{j} \approx \vec{S}_{j}^{(0)}$. For the first time step $t_{1}$, we let $\vec{S}_{j}^{(0)}=\vec{S}_{j-1}$, and for subsequent time steps $(j>1), \vec{S}_{j}^{(0)}$ is interpolated from the previous two time steps such that $\vec{S}_{j}^{(0)}=2 \vec{S}_{j-1}-\vec{S}_{j-2}$. The solution is then updated in subsequent iterations using Newton's Method

$$
\begin{aligned}
\vec{S}_{j}^{(k+1)} & =\vec{S}_{j}^{(k)}-\left[\vec{F}^{\prime}\left(\vec{S}_{j}^{(k)}\right)\right]^{-1} \vec{F}\left(\vec{S}_{j}^{(k)}\right), \\
& =\vec{S}_{j}^{(k)}+\vec{H}^{(k)},
\end{aligned}
$$

where $\vec{F}^{\prime}\left(\vec{S}_{j}^{(k)}\right)$ is the Jacobian matrix of $\vec{F}$ evaluated at $\vec{S}_{j}^{(k)}$ and $\vec{H}^{(k)}$ is the correction to the approximated solution that was computed at the previous iteration $\vec{S}_{j}^{(k)}[13]$. Computing $\vec{S}_{j}^{(k+1)}$ in $(7)$ requires solving the linear system

$$
\vec{F}^{\prime}\left(\vec{S}_{j}^{(k)}\right) \vec{H}^{(k)}=-\vec{F}\left(\vec{S}_{j}^{(k)}\right)
$$

for $\vec{H}^{(k)}$. Since the $i^{t h}$ equation in the nonlinear system (6) depends only on the unknowns $S_{i+2, j}, S_{i+1, j}, S_{i, j}, S_{i-1, j}$, and $S_{i-2, j}$ for $2 \leq i \leq N$, that is

$$
F_{i}\left(0, \cdots, 0, S_{i-2, j}, S_{i-1, j}, S_{i, j}, S_{i+1, j}, S_{i+2, j}, 0, \cdots, 0\right)=0
$$

and since

$$
\begin{aligned}
F_{1} & =S_{1, j}-1=0, \\
F_{N+1} & =S_{N+1, j}-\alpha-0.1 \frac{h_{0}}{r_{0}}=0,
\end{aligned}
$$

Eq. (8) is a pentadiagonal linear system. We iterate Newton's method at each time step until the $l_{\infty}$-norm of $\vec{H}^{(k)}$ is sufficiently small, that is,

$$
\left\|\vec{H}^{(k)}\right\|_{\infty}=\max _{1 \leq i \leq N+1}\left|H_{i}^{(k)}\right| \leq 10^{-12}
$$

where $\vec{H}^{(k)}=\left[H_{1}^{(k)}, H_{2}^{(k)}, \cdots, H_{N+1}^{(k)}\right]$ which corresponds to using four Newton iterations at each time step in the numerical simulations.

We developed our own Fortran code to implement the numerical methods described above. In particular, to perform Newton's iterative method at each time step, code was written to compute the Jacobian matrix $\vec{F}^{\prime}\left(\vec{S}_{j}^{(k)}\right)$ and the vector $\vec{F}\left(\vec{S}_{j}^{(k)}\right)$ in (8). Given the pentadiagonal structure of the Jacobian matrix, we used the routines bandec and banbks from Numerical Recipes [14] to solve the banded diagonal system (8) for $\vec{H}^{(k)}$, rather than LU factorization 
TABLE I: Experimental conditions for castor oil with $r_{f}=0.029 \mathrm{~cm}$ [3]. The values listed below were used in the numerical simulations.

\begin{tabular}{ccccc}
\hline \hline Data Set & $\begin{array}{c}r_{0} \\
(\mathrm{~cm})\end{array}$ & $\alpha=r_{f} / r_{0}$ & $\epsilon=\left(\rho g r_{0}^{2}\right) / \gamma$ & $\begin{array}{c}\mathrm{Q} \\
\left(\mathrm{cm}^{3} / \mathrm{s}\right)\end{array}$ \\
\hline 1 & 0.1123 & 0.2582 & 0.316 & 0.0194 \\
2 & 0.1107 & 0.2620 & 0.307 & 0.0171 \\
3 & 0.1083 & 0.2678 & 0.294 & 0.0148 \\
4 & 0.1054 & 0.2751 & 0.278 & 0.0123 \\
5 & 0.1039 & 0.2791 & 0.270 & 0.0111 \\
6 & 0.1026 & 0.2827 & 0.264 & 0.0103 \\
\hline \hline
\end{tabular}

which is computationally less efficient.

The free boundary problem (4) and (5) requires three physical parameters $r_{0}, \alpha$, and $\epsilon$. Values for these parameters are taken directly from Smolka, North and Guerra's experiments with castor oil and are reported in Table I [3]. In their experiments, the surface tension, density, and viscosity of castor oil are $\gamma=36.8$ dyne $/ \mathrm{cm}^{2}, \rho=0.94 \mathrm{~g} / \mathrm{cm}^{3}$, and $\mu=8.48 \mathrm{~g} /(\mathrm{cm} \cdot \mathrm{s})$, respectively, and the fiber radius is $r_{f}=0.029 \mathrm{~cm}$. SNG present six data sets in their castor oil experiments corresponding to values of $r_{0}$ ranging between 0.1026 and $0.1123 \mathrm{~cm}$. In their paper these data sets are identified using the flow rate of fluid from the orifice, $Q$, which for convenience is also reported in Table I; the flow rate and unperturbed radial distance to the free surface, $r_{0}$, are related by the formula [3]

$$
Q\left(r_{f}, r_{0}\right)=\frac{\rho g \pi}{8 \mu}\left[4 r_{f}^{2} r_{0}^{2}+4 r_{0}^{4} \ln \left(r_{0} / r_{f}\right)-r_{f}^{4}-3 r_{0}^{4}\right] .
$$

Given the above parameters, the Reynolds and Bond numbers in their experiments ranged between $0.049 \leq \operatorname{Re} \leq 0.054$ and $0.26 \leq \epsilon \leq 0.32$, respectively, which confirms that these experiments satisfy the requirements of Craster and Matar's Stokes flow model $(\operatorname{Re} \leq O(1), \epsilon \ll 1)$ [3]. In our numerical study, we will conduct simulations using the parameters from these six data sets.

In dimensional units, the domain on which we numerically solve (4) and (5) corresponds to a $1 \mathrm{~m}$ long fiber (that is, $X=100 \mathrm{~cm}$ ); the experiments conducted by SNG used a $2 \mathrm{~m}$ long fiber [3]. In all of the simulations we use spatial step length $d z \approx 0.0029 \mathrm{~cm}$ (corresponding to 35,000 axial grid points) and time step length $d t \approx 0.00026 \mathrm{~s}$. To ensure that the size of $d z$ and $d t$ did not influence the numerical results, we conducted simulations with smaller values of these parameters and found indistinguishable results from the ones presented here.

\section{OBSERVATIONS FROM THE NUMERICAL SIMULATIONS}

\section{A. General Observations}

Figure 7 shows the evolution of the free surface near the top of the fiber at the onset of the simulations with $r_{0}=0.1123 \mathrm{~cm}, \alpha=0.2582$, and $\epsilon=0.316$ corresponding to data set 1 in Table I. In this figure the top of the fiber is located at $z=0$ and the axes are not drawn to scale. The contact line is initially located $4.9 \mathrm{~cm}$ from the top of the fiber (see Fig. 7(a)). After $2.6 \mathrm{~s}$ (Fig. 7(b)), a capillary ridge forms behind the contact line, denoted by the large bump, as the contact line travels down the fiber; behind this capillary ridge, the free surface decays in a damped oscillation. As the contact line continues to travel down the fiber, infinitesimal oscillations of the free surface not visible to the eye persist in the region where the contact line was initially located at $z \sim 5 \mathrm{~cm}$ (Fig. 7(c)-(e)). The oscillations, which represent perturbations of the free surface, are convected down the fiber growing in amplitude and then saturating in size as they trail behind the contact line.

Figure 8(a) shows a snapshot of the free surface after $142.81 \mathrm{~s}$ have elapsed in the simulation from Figure 7(a); the axes are not drawn to scale. The flow has now almost completely covered the fiber with the contact line $\sim 85 \mathrm{~cm}$ down the fiber and with 92 perturbations trailing behind it. The variation in the perturbation amplitude, here about $4.4 \%$, was typical in the simulations of all six data sets. The actual shape of the perturbations are shown in Fig. 8(b) which represents a zoomed in section of the free surface with the radial and axial distances drawn to scale; the image was produced by reflecting Fig. 8(a) across the fiber axis. The arrows in the figure indicate the axial location of perturbation maxima not visible to the eye. We find the perturbations are first detectable at $z \sim 10 \mathrm{~cm}$ which is approximately $5 \mathrm{~cm}$ further down the fiber than at the beginning of the simulation; this can be seen by comparing Figs. 7 and 8. In the castor oil experiments, the axial position where perturbations were first detectable did not 

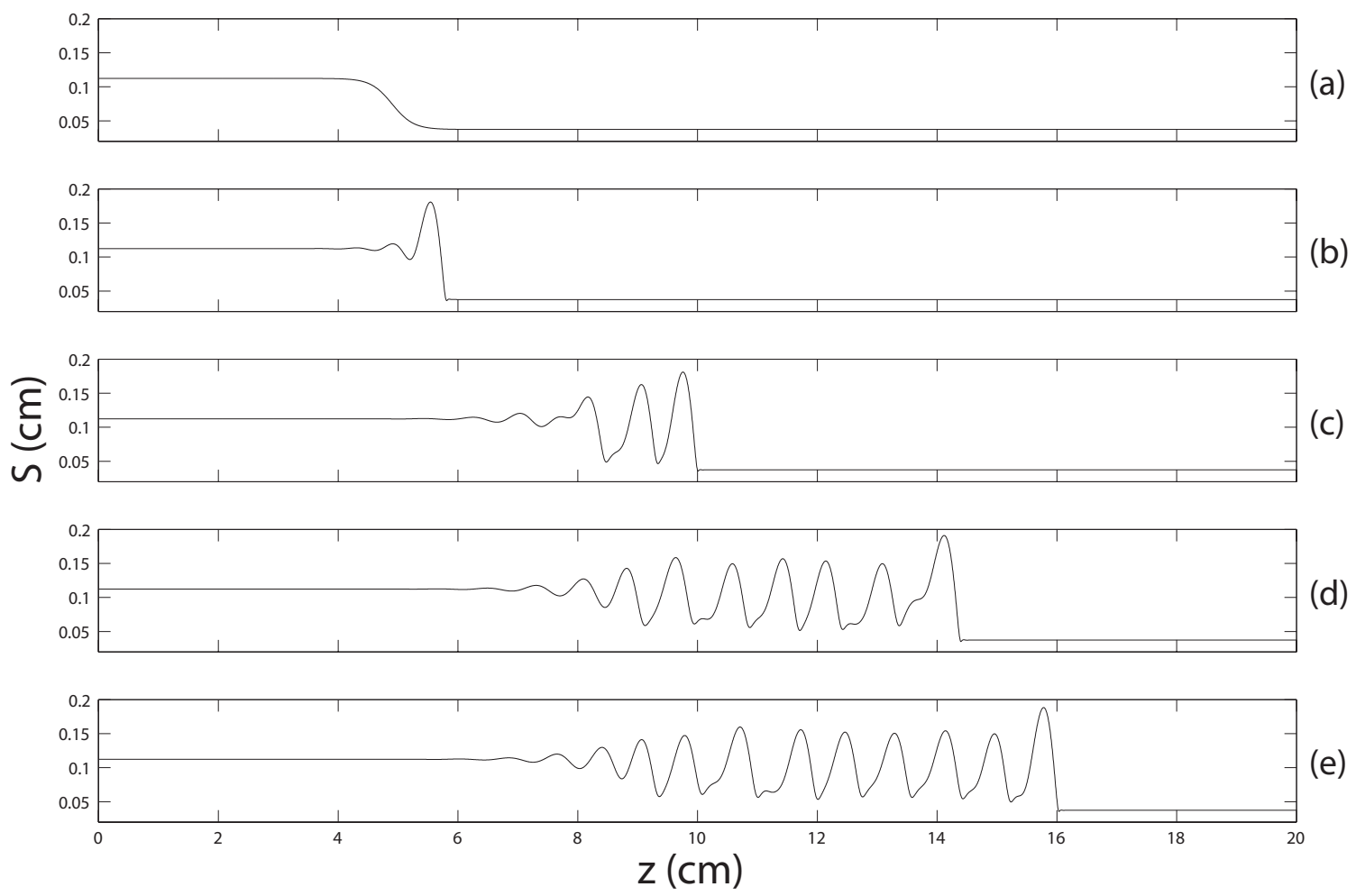

FIG. 7: A sequence of images showing the evolution of the free surface at the onset of the simulations with $r_{0}=0.1123 \mathrm{~cm}$, $\alpha=0.2582$, and $\epsilon=0.316$, corresponding to data set 1 in Table I. The oscillations in the free surface correspond to individual perturbations. Image times, referenced from the beginning of the simulations, are: (a) $t=0 \mathrm{~s},(\mathrm{~b}) t=2.60 \mathrm{~s}$, (c) $t=10.39 \mathrm{~s}$, (d) $t=18.17 \mathrm{~s}$, (e) $t=20.77 \mathrm{~s}$. The top of the fiber is located at $z=0 \mathrm{~cm}$. The graphs are rotated $90^{\circ}$ counterclockwise from the physical set-up.

change in time for a fixed value of $r_{0}$ or $Q$ [3], whereas in all of our simulations, we find the axial position drifts down the fiber. To replicate the experimental conditions as close as possible in the simulations, we chose the initial location of the contact line (at $t=0$ in the simulations) to be at the axial position where perturbations were first detectable in the experiments.

In the castor oil experiments, SNG found that when the flow rate exceeded the critical value, $Q_{c}=0.0095 \mathrm{~cm}^{3} / \mathrm{s}$, neighboring perturbations could coalesce, but only after their amplitude saturated in size [3]. SNG referred to this as unsteady behavior [3]. As an example, the sequence of images in Fig. 2 shows that no coalescence occurs while several perturbations grow in amplitude even though $Q>Q_{c}$ in this experiment (the experimental parameters correspond to data set 1). This behavior was typical in all of the castor oil experiments. We observe much different behavior in the simulations. Figure 9 represents a continuation of the simulation begun in Fig. 7(a) with the real time elapsed by $165.24 \mathrm{~s}$. The physical parameters used in the simulation, corresponding to data set 1 , match those of the experimental images and data in Figs. 2 and 3. In Fig. 9 the axial position of perturbation maxima is tracked for five perturbations, labelled 1 through 5 in the figure, but which we shall refer to as P1 through P5. In Fig. 9(a)-(e) the distance between P4 and P5 decreases as each perturbation grows in amplitude. By Fig. 9(f) P4 and P5 have completely coalesced. Similarly, while P1 and P2 grow in amplitude in Fig. 9(b)-(g), the distance between them decreases and the perturbations coalesce by Fig. 9(h). In the simulations most perturbations coalesced with their neighbor while growing in size, whereas in the experiments no coalescence was observed. The early time spatiotemporal behavior of the perturbations predicted by the CM model are quite different than the experimental observations of SNG. Next, we investigate how interactions between neighboring perturbations in the simulations influence the initial growth of perturbations. 

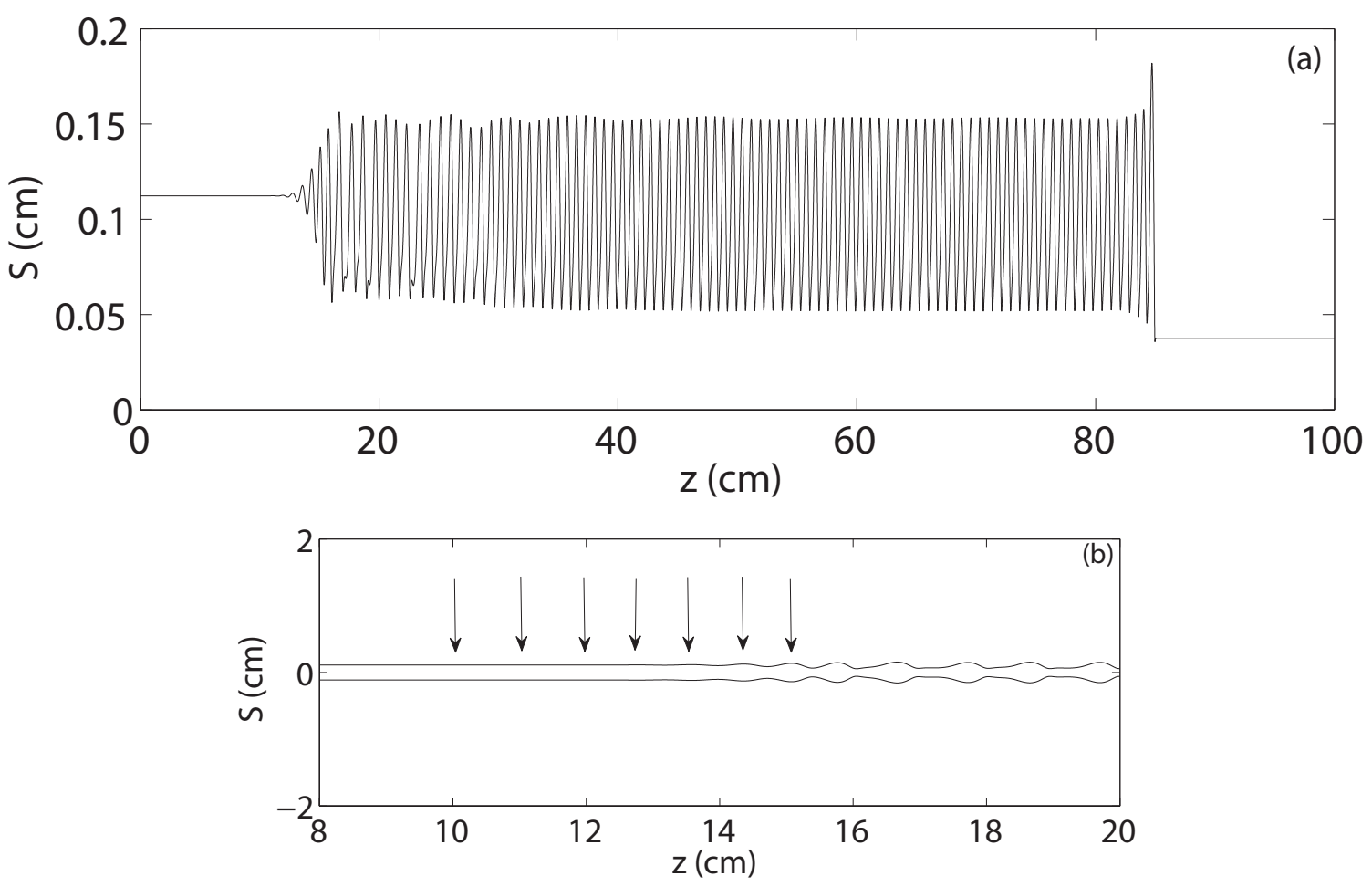

FIG. 8: (a) A snapshot in time of the free surface with $r_{0}=0.1123 \mathrm{~cm}, \alpha=0.2582$, and $\epsilon=0.316$ corresponding to data set 1 in Table I with time elapsed by $142.81 \mathrm{~s}$ from Fig.7(a). Oscillations in the free surface correspond to individual perturbations. Image not drawn to scale. (b) A zoomed in section of the flow in the region where perturbations first appear along the free surface in (a); the data from (a) has been reflected across the $z$-axis and arrows indicate the location of perturbation maxima. The axes in (b) have an equal aspect ratio. The top of the fiber is at $z=0 \mathrm{~cm}$ and the bottom of the fiber is at $z=100 \mathrm{~cm}$.

\section{B. Perturbation Formation}

Next we compare the growth of perturbations in the experiments and simulations. Following SNG [3], we measure the perturbation amplitude, $\eta$, as half the radial distance from the first minima to the first maxima and the wavelength, $\lambda$, as the axial distance from the first to second maxima as shown in the schematic of the flow in Fig. 4 . We have already observed in the simulations that perturbations display much different spatiotemporal behavior than in the experiments (Fig. 9). In order to compare the perturbation growth in experiments and simulations, we track perturbations in the simulations that do not coalesce with their neighbors. We see in Fig. 9 that perturbation P3 satisfies this requirement and so we will focus on this perturbation's growth. To start, we'll summarize the experimental results of SNG, and then present results from our simulations.

The experimental data shown in Fig. 3 for perturbation amplitude and wavelength, which corresponds to the perturbation tracked in Fig. 2, represents typical behavior observed in the castor oil experiments of SNG [3]. Namely, the perturbation amplitude initially grows exponentially, and then slows over time until the amplitude saturates in size as shown in Fig. 3(a); the data forms an S-shaped curve with one inflection point that is reminiscent of the logistic curve. The wavelength decreases during the time interval that the amplitude grows exponentially and then remains constant as the amplitude growth slows down, as shown in Fig. 3(b). The time period, growth rate, amplitude and wavelength during the exponential phase of amplitude growth and the saturated amplitude are listed in Table II.

Figure 10 shows measurements of the amplitude (on linear and log-linear scales) and the wavelength of P3 with $t=0$ referenced from Fig. 9(a). We find the amplitude growth has two inflection points, instead of one as in the experiment, before saturating in size (see Fig. 10(a)). In Fig. 10(b) we identify three phases to the perturbation's growth. In the first two phases the amplitude grows exponentially though at different rates and during the third phase the amplitude saturates in size. The time period, growth rate, amplitude and wavelength during each phase and the saturated amplitude are listed in Table II. We find that a perturbation can be detected earlier in the simulations than in the experiments which allows for smaller amplitudes to be measured in the simulations. During phase I, the value of $\eta$ is between one and two orders of magnitude smaller and $\sigma$ is larger than experimental values, whereas during 


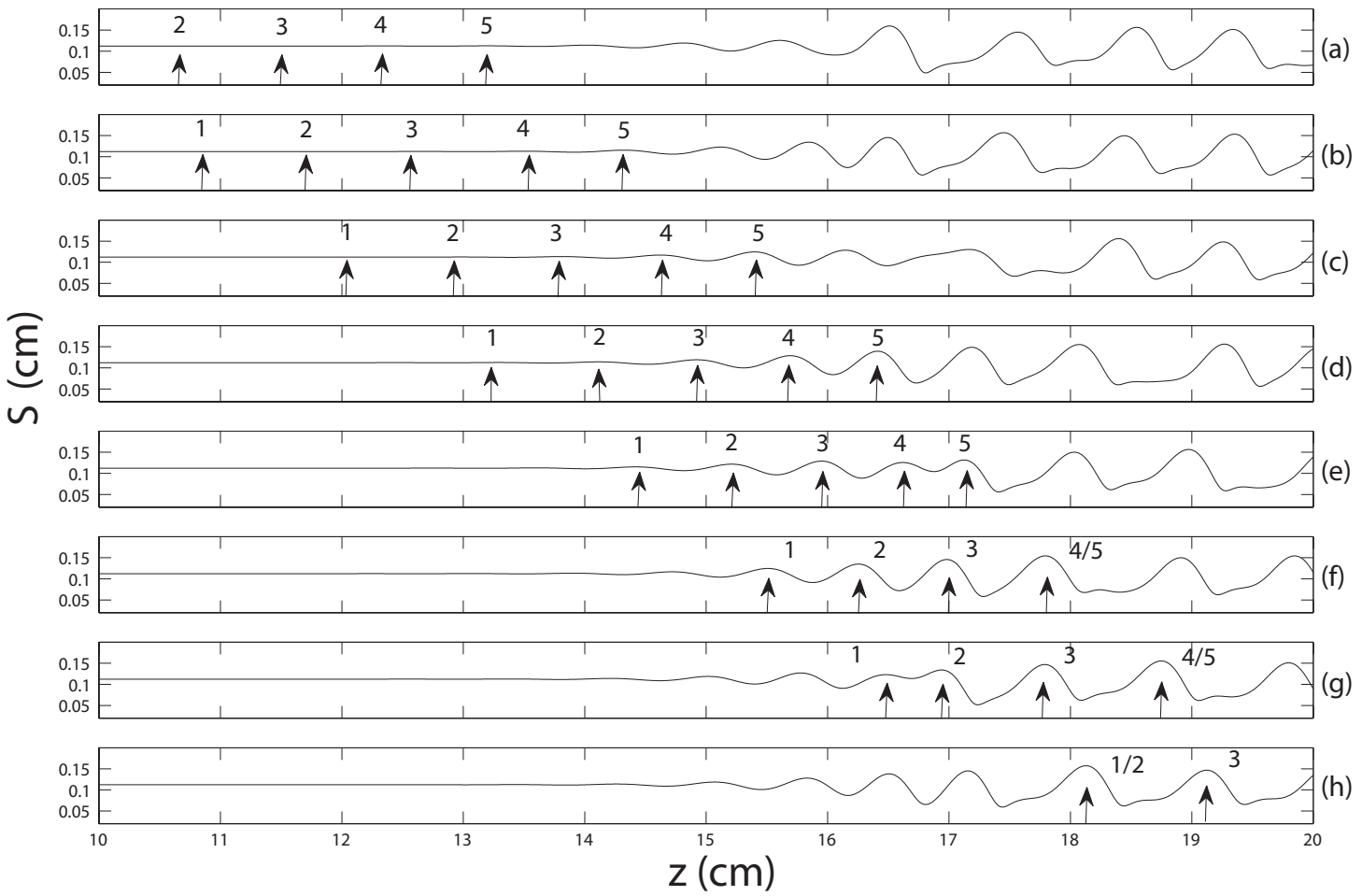

FIG. 9: A sequence of images from simulations showing the dynamics of several neighboring perturbations with $r_{0}=0.1123 \mathrm{~cm}$, $\alpha=0.2582$, and $\epsilon=0.316$ corresponding to data set 1 . This figure represents a continuation of the simulation begun in Fig. 7 (a) with the time elapsed by $165.24 \mathrm{~s}$. The arrows indicate the axial location of perturbation maxima. As the amplitude of P3 increases, the perturbations in front of P3 (P4 and P5) and behind P3 (P1 and P2) get closer and eventually coalesce; P4 and P5 coalesce at $\sim 4.2 \mathrm{~s}$ and $\mathrm{P} 1$ and P2 coalesce at $\sim 6.4 \mathrm{~s}$. Image times relative to (a) are: (a) $t=0 \mathrm{~s},(\mathrm{~b}) t=1.04 \mathrm{~s},(\mathrm{c})$ $t=2.08 \mathrm{~s}$, (d) $t=3.12 \mathrm{~s}$, (e) $t=4.16 \mathrm{~s}$, (f) $t=5.20 \mathrm{~s}$, (g) $t=6.24 \mathrm{~s}$, (h) $t=7.28 \mathrm{~s}$.

TABLE II: A comparison of experimental and simulation data with $r_{0}=0.1123 \mathrm{~cm}, \alpha=0.2582$, and $\epsilon=0.316$ corresponding to the physical parameters of data set 1 . The experimental data corresponds to the perturbation tracked in Figs. 2 and 3 and the simulation data corresponds to perturbation P3 shown in Figs. 9 and 10. The value $\Delta t$ is the time period of exponential growth of the amplitude, $\sigma, \eta$ and $\lambda$ are the values of the growth rate, amplitude and wavelength during time period $\Delta t$ and $\eta_{\text {saturate }}$ is the amplitude of the perturbation once it saturates in size.

\begin{tabular}{|c|c|c|c|c|c|}
\hline & $\begin{array}{l}\Delta t \\
(\mathrm{~s})\end{array}$ & $\begin{array}{c}\sigma \\
\left(\mathrm{s}^{-1}\right)\end{array}$ & $\begin{array}{c}\eta \\
(\mathrm{mm})\end{array}$ & $\begin{array}{c}\lambda \\
(\mathrm{cm})\end{array}$ & $\begin{array}{c}\eta_{\text {saturate }} \\
\text { (mm) }\end{array}$ \\
\hline Experiment & 2.3 & 0.935 & $0.025-0.19$ & $0.82-0.98$ & 0.35 \\
\hline Simulation Phase I & 3.1 & 2.03 & $1.1 \mathrm{e}-4-0.05$ & $0.77-0.83$ & - \\
\hline Simulation Phase II & 2.5 & 0.741 & $0.05-0.45$ & $0.67-1.06$ & - \\
\hline Simulation Phase III & - & - & - & $0.93-0.99$ & 0.48 \\
\hline
\end{tabular}

phase II these values are more comparable to those in the experiment. In the third phase, the saturation size of the amplitude in the simulation is $37 \%$ larger than in the experiment. We found in our simulations of all six data sets that the saturation size ranged between $37 \%$ and $42 \%$ larger than the experimental values. As for the wavelength, the trend of the data is different in the simulations than in the experiment (see Fig. 3(b)), however, the general range of values of $\lambda$ predicted by the simulation is comparable to the experimental values.

Clearly the behaviors for the amplitude and wavelength predicted by Craster and Matar's model in the simulation are quite different than what is observed in the experiments. A key difference between the perturbation tracked in the experiment, shown in Fig. 2, and P3 in the simulation is in the dynamics of neighboring perturbations. In the experiment, no coalescence occurs while the perturbation grows, as shown in Fig. 2. By contrast in the simulation, the two neighboring perturbations in front of P3 (P4 and P5) and behind P3 (P1 and P2) coalesce while P3 grows in amplitude. We investigate the influence of these interactions on the growth of P3 more closely by examining the 

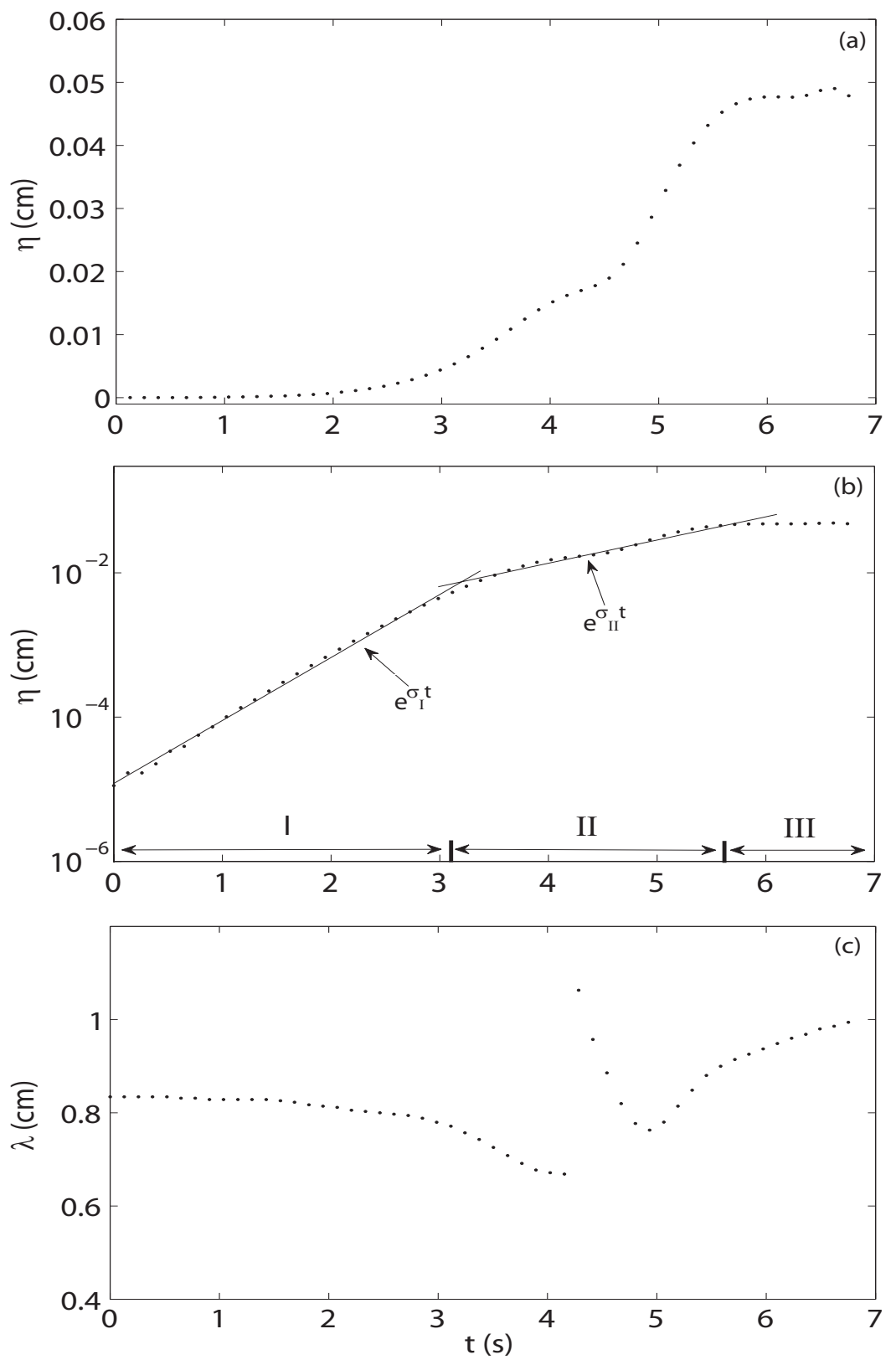

FIG. 10: Simulation results of amplitude vs. time plotted on (a) a linear scale, (b) a log-linear scale, and of (c) wavelength vs. time of P3 (shown in Fig. 9). The growth of the amplitude follows three distinct phases as indicated in (b). There are two distinct phases of exponential growth indicated by the solid lines in (b); best fits of the data are: $\eta_{I}(t)=0.00001 e^{2.011 t}(\mathrm{phase}$ I) and $\eta_{I I}(t)=0.0007 e^{0.741 t}$ (phase II). Phase III corresponds to saturation of the amplitude. The time intervals for Phases I, II and III are: $0 \leq t \leq 3.1 \mathrm{~s}, 3.1<t \leq 5.6 \mathrm{~s}$ and $t>5.6 \mathrm{~s}$, respectively. Simulations use the parameters from data set 1 (Table I). Time $t=0 \mathrm{~s}$ is referenced from the simulation data in Fig. 9(a).

early time behavior of perturbations $\mathrm{P} 1, \mathrm{P} 2, \mathrm{P} 4$ and $\mathrm{P} 5$.

We have already noted that P4 and P5 move closer together and then coalesce between Fig. 9(a)-(f) and that P1 and P2 move closer together and then coalesce between Fig. 9(c)-(h). To quantify this behavior, we plot the wavelength between P4 and P5 as a function of time in Fig. 11. Here we find that the speed P4 and P5 move towards each other, indicated by the slope of the data, increases at $t=3.1 \mathrm{~s}$ which corresponds in time to Fig. 9(d). Fig. 11 also shows that $\mathrm{P} 4$ and $\mathrm{P} 5$ coalesce at $t=4.2 \mathrm{~s}$ as indicated by the data point at $\lambda=0$. We find $\mathrm{P} 1$ and $\mathrm{P} 2$ demonstrate similar behavior with the speed that they approach each other increasing at $t=5.3 \mathrm{~s}$ (corresponding approximately to Fig. 9(f)) and coalesce at $t=6.4 \mathrm{~s}$; for brevity a plot of the wavelength between P1 and P2 as a function of time is not shown. 


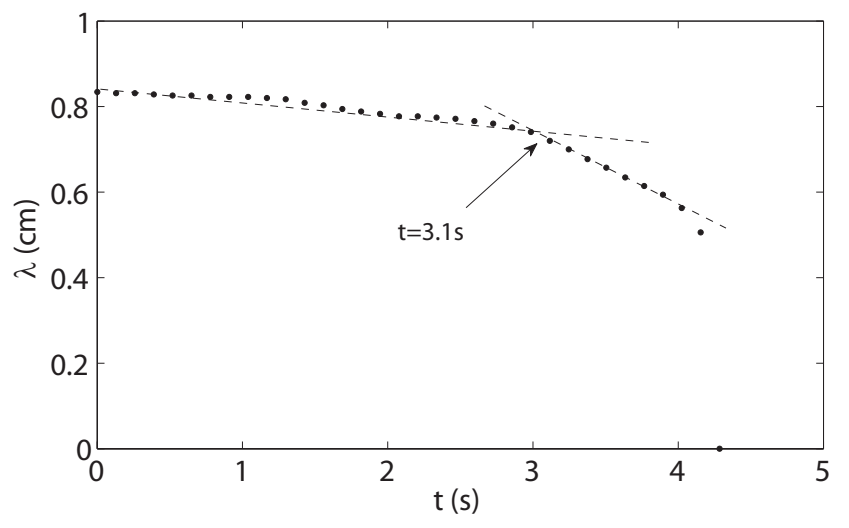

FIG. 11: Wavelength vs. time measured between perturbations P4 and P5 (shown in Fig. 9). The speed that P4 and P5 move towards each other, approximated by the slope of the dashed lines, increases at $t=3.1 \mathrm{~s}$. The perturbations coalesce at $t=4.2 \mathrm{~s}$ since $\lambda=0$. Time $t=0 \mathrm{~s}$ is referenced from the simulation data in Fig. $9(\mathrm{a})$.

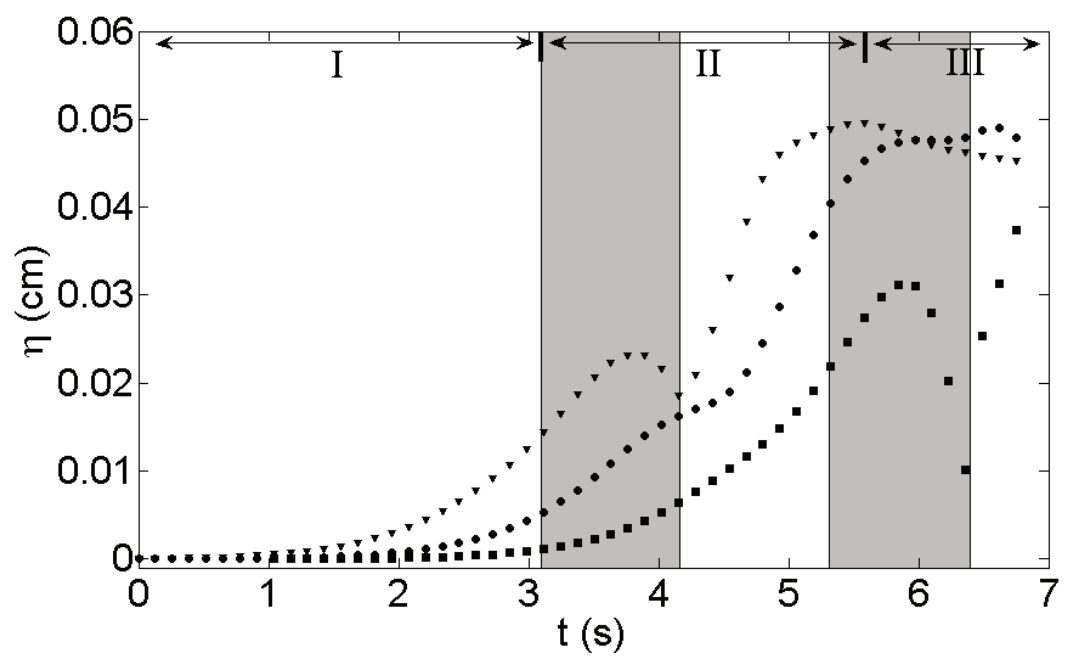

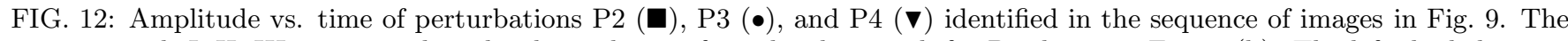
time intervals I, II, III correspond to the three phases of amplitude growth for P3 shown in Fig. 10(b). The left-shaded region corresponds to the time period when P4 and P5 move towards each other more rapidly and coalesce and the right-shaded region corresponds to the time period when P1 and P2 move towards each other more rapidly and coalesce (see Fig. 9).

To understand how the dynamics of the perturbations in front of and behind P3 influence it's growth, we show the amplitude as a function of time for P2 $(\boldsymbol{\nabla})$, P3 $(\bullet)$, and P4 $(\boldsymbol{\nabla})$ in Fig. 12. The left-shaded region indicates the time period $(3.1 \leq t \leq 4.2 \mathrm{~s})$ that $\mathrm{P} 4$ and $\mathrm{P} 5$ move towards each other more rapidly. At $t=4.2 \mathrm{~s}, \mathrm{P} 4$ has merged with $\mathrm{P} 5$ and the subsequent data $(\mathbf{\nabla})$ represents the amplitude of the coalesced perturbation. A consequence of P4 and P5 merging is that the wavelength of P3 sharply increases at $t=4.2 \mathrm{~s}$ (see Fig. 10(c)). More importantly, the onset of this time period corresponds exactly to the time when the exponential growth rate of the amplitude of P3 transitions from $\sigma_{I}$ to $\sigma_{I I}$ (shown in Fig. 10(b)). This provides evidence that the transition in the rate of growth of the amplitude of P3 is influenced by the dynamics of the perturbations in front of it. The right-shaded region indicates the time period $(5.3 \leq t \leq 6.4 \mathrm{~s})$ that $\mathrm{P} 1$ and $\mathrm{P} 2$ move towards each other more rapidly. This time period roughly corresponds to phase III when the amplitude growth of P3 slows down and the amplitude saturates in size. At $t=6.4 \mathrm{~s}, \mathrm{P} 1$ has merged with P2 and the subsequent data $(\mathbf{\square})$ represents the amplitude of the coalesced perturbation.

Why does the exponential growth rate for the amplitude of P3 change at $t=3.1 \mathrm{~s}$ ? While answering this question requires a more detailed study, we provide one possible explanation. For a perturbation to grow in size, nearby fluid must move into the perturbation in order to conserve mass. It is well established that fluid flows from high to low pressure regions [15]. For a free surface flow, fluid pressure is proportional to the curvature of the free surface. In 
Fig. 9(a)-(c), the curvatures of P3 and P4 are nearly equal, thus we estimate that the fluid pressures inside P3 and P4 are nearly equal. Since the attenuated region between P3 and P4 has a higher curvature, fluid should move from this region into P3 and P4. If it is true that the fluid pressures in P3 and P4 are nearly equal, then fluid should move into these perturbations from the attenuated region at nearly the same rate (ignoring other effects such as gravity). At the time that P4 and P5 move more quickly towards each other $(t=3.1 \mathrm{~s}$, Fig. $10(\mathrm{~d}))$, the curvatures of P3 and $\mathrm{P} 4$ are no longer equal. We conjecture that fluid in $\mathrm{P} 4$ has lower pressure than in P3 which causes the fluid in the attenuated region to preferentially move into $\mathrm{P} 4$ rather than P3, and it is this effect that leads to a slow down in the growth of the amplitude of P3. To check this assertion requires a more detailed analysis of the local curvatures and pressures in the flow which we leave for a future study.

Finally, we note that the behaviors shown in Figs. 10-12 were universal in the simulations that we conducted for all six data sets. In particular, we were unable to identify a sequence of neighboring perturbations that did not interact and coalesce during their initial formation. This is in contrast to the behavior observed in the experiments in which no coalescence was observed between neighboring perturbations during the same time period [3]. We are unable to explain why the perturbations in the simulations coalesce so early in their development and instead leave this question for a future study. A numerical study of Craster and Matar's model by Ruyer-Quil et al. [6] has found discrepancies in the spatiotemporal dynamics predicted by the CM model $[6,16]$ which may provide some insight into the discrepancies we observe in our numerical study.

\section{Comparison of Growth Rates}

Next we focus on the stage of a perturbation's formation when the amplitude grows exponentially by comparing numerical results to experimental data for all six data sets; the experimental data appears in [3] (see their Fig. 6(a)). Figure 13 shows a comparison of the amplitude growth rate measured in the simulations and in the experiments and the dispersion curve developed from linear stability analysis by Craster and Matar (2b). Figure 13(a) corresponds to the experimental conditions for data set 1, Figure 13(b) corresponds to data set 2, and so forth, with each data set corresponding to a different experimental flow rate $Q$ or $r_{0}$ (see Table I). In the plots, the average dimensionless growth rate $\left(\hat{\sigma}=\sigma_{\operatorname{dim}} \mathcal{L} / \mathcal{V}\right)$ and dimensionless wavenumber $(\hat{m}=2 \pi \mathcal{L} / \lambda)$ measured over 10 perturbations in a simulation are denoted by a square for phase I (only in Fig. 13(a)) and a circle for phase II and the vertical bars represent the standard deviation of those growth rates. Since the perturbation wavelength varies during the exponential phases of growth in the simulation, we cannot assign a single wavenumber to it's growth. Instead, the horizontal bars represent the range of wavenumber during the period of exponential growth in phase I or phase II, respectively, of all the perturbations measured. As an aside, we found that neither the location of the contact line as it moves down the fiber nor the specific length of the fiber influenced the values of the growth rate in the simulations. The average dimensionless growth rate and dimensionless wavenumber measured over 8-12 perturbations in an experiment are denoted by a triangle, the vertical bars represent the standard deviation of those growth rates, and the horizontal bars represent the range of wavenumber during the period of exponential growth of all the perturbations measured in the experiments [3]. We find in all six data sets that the growth rates measured during phase I significantly overestimate the experimental values; Fig. 13(a) shows the most favorable comparison. Since the perturbation amplitude during phase I is one to two orders of magnitude smaller than in the experiments (see Table II), we choose not to compare growth rates during this phase. However, the amplitude in phase II of the simulations is the same order of magnitude as in the experiments, so we will compare data from phase II to experimental data. Of the six data sets, we find excellent agreement between the numerical results during phase II and the experimental data in two of the data sets (data sets 1 and 3, corresponding to Figs. 13(a) and 13(c)) in that the growth rates are equal within the resolution of the data and the range of wavenumber are consistent. The agreement for two other sets is fair to good (data sets 2 and 4 , corresponding to Figs. 13(b) and 13(d)). At the lowest two flow rates, the growth rates are significantly different and there is little overlap in the range of wavenumber between the numerical results and the experimental data (data sets 5 and 6, corresponding to Figs. 13(e) and 13(f)). Recall that in the simulations neighboring perturbations coalesced while a perturbation formed, whereas in the experiments no such coalescence occurred [3]; this may account for the uneven results. It is noteworthy that we find some agreement between the numerical results and the experimental data given these differences in the perturbation dynamics. We also compare the growth rates measured in the simulations to predictions from the linear stability theory developed by Craster and Matar (2b) [2]. The dispersion curves represent the real part of the growth rate given by $(2 \mathrm{~b})$ plotted over the range of wavenumber measured in both the simulation and the experiment. We consider the theory to be in qualitative agreement with the numerical results, at a given flow rate, if the theoretical curve overlaps with the rectangular region defined by the resolution bars of the numerical data. Figure 13 shows that the linear stability theory is in excellent qualitative agreement with the numerical data collected during phase II for all six data sets. This is a significant result considering that the comparison is between a temporal stability theory and a spatial instability of the free surface. Finally, we note that the growth rates measured 

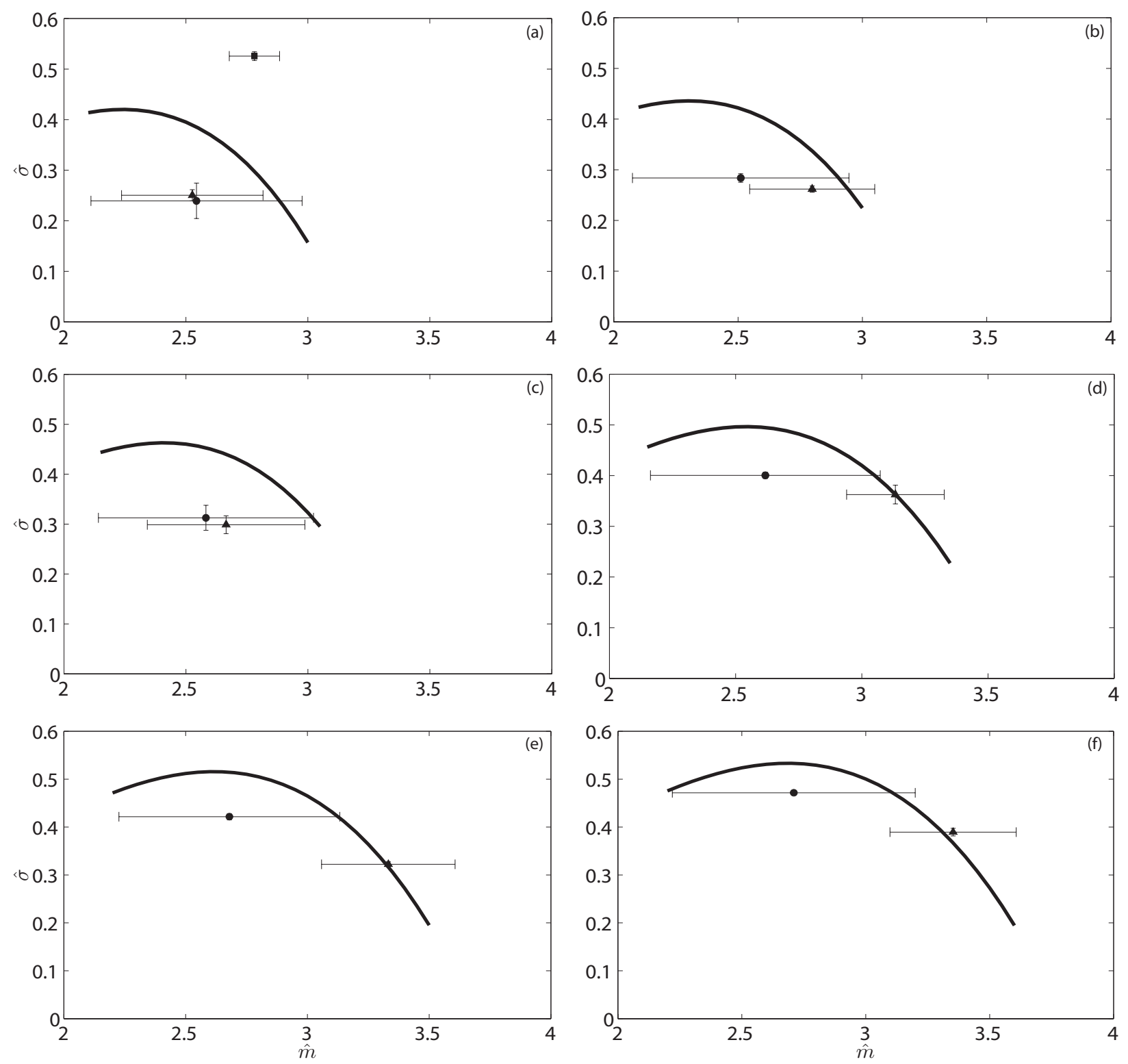

FIG. 13: Dimensionless growth rate as a function of dimensionless wavenumber in simulations and experiments. Figures correspond to: (a) data set $1\left(Q=0.0194 \mathrm{~cm}^{3} / \mathrm{s}\right),(\mathrm{b})$ data set $2\left(Q=0.0171 \mathrm{~cm}^{3} / \mathrm{s}\right)$, (c) data set $3(Q=0.0148 \mathrm{~cm} / \mathrm{s}),(\mathrm{d})$ data set $4\left(Q=0.0123 \mathrm{~cm}^{3} / \mathrm{s}\right)$, (e) data set $5\left(Q=0.0111 \mathrm{~cm}^{3} / \mathrm{s}\right)$, and (f) data set $6\left(Q=0.0103 \mathrm{~cm}^{3} / \mathrm{s}\right)$. Circles correspond to numerical data for the average growth rate and wavenumber measured over 10 perturbations during phase II in each data set. Vertical bars represent the standard deviation of $\hat{\sigma}$ and horizontal bars represent the range of $\hat{m}$ measured during the period of exponential growth in phase II over all perturbations measured. Triangles correspond to experimental data measured over 8-12 perturbations, vertical bars represent the standard deviation of $\hat{\sigma}$, and horizontal bars represent the range of $\hat{m}$ measured during the period of exponential growth over all perturbations measured [3]. Corresponding curves represent the real part of $\hat{\sigma}$ predicted by linear stability theory using CM's model (2b), plotted over the range of $\hat{m}$ measured in both the simulations and experiments. The square in (a) indicates the average growth rate and wavenumber measured over 10 perturbations during phase I and the vertical and horizontal bars represent the standard deviation of $\hat{\sigma}$ and the range of $\hat{m}$ measured during the period of exponential growth in phase I over all perturbations measured. In all six data sets, the growth rate during phase I is significantly larger than the experimental data and the values predicted by CM's model; (a) shows the most favorable comparison. 
during phase I are significantly larger than those predicted by linear stability theory (between a factor of 1.5 and 3.0 larger) in the six data sets.

\section{CONCLUSIONS}

We numerically solve a fourth order nonlinear partial differential equation derived by Craster and Matar [2] that models a viscous fluid flowing down the outside of a vertical fiber in order to investigate the initial formation of perturbations along the fluid free surface. We compare observations from numerical simulations of the CM model to experimental data collected by Smolka, North, and Guerra [3]; six data sets corresponding to different experimental conditions are tested. We find that in the simulations for all six data sets, perturbations consistently coalesced with neighboring perturbations during their initial formation. In contrast, no coalescence was observed during the same time period in the experiments [3].

The dependence of perturbation amplitude on time in the simulations does not follow an S-shaped curve as in the experiments. The amplitude in the simulations follows two distinct exponential functions (which we refer to as phases I and II) in the early time growth of the perturbation; in the experiments the data follows only one exponential function. The values of the amplitude during phase I are between one and two orders of magnitude smaller than those measured in experiments, whereas the values in phase II are the same order of magnitude as in the experiments. The growth rates during phase I are up to a factor of 2.6 times larger than those in phase II. We find the switch in growth rates from phase I to II appears to be influenced by the interaction of the two perturbations in front of the perturbation of interest. Data from simulations of Craster and Matar's model consistently overpredict the final perturbation amplitude compared to experimental data.

We compare the growth rates of several perturbations during phase II from simulations of the CM model to experimental data. Of the six data sets, the range of growth rate and wavenumber were in excellent agreement in two of the sets and in fair to good agreement with two other sets. For the last two sets, the growth rates and range of wavenumber were significantly different. The coalescence of neighboring perturbations observed in the simulations of Craster and Matar's model may be the cause of the discrepancy in the growth rates and wavelengths. We find that linear stability results developed from Craster and Matar's Stokes flow model [2] are in excellent qualitative agreement with the growth of the perturbations during phase II measured in simulations for all six data sets.

\section{Acknowledgments}

The authors acknowledge support from NSF Grant No. DMS-0707755.

[1] I. L. Kliakhandler, S. H. Davis, and S. G. Bankoff, Viscous beads on vertical fibre, J. Fluid Mech. 429 (2001), pp. 381-390.

[2] R. V. Craster and O. K. Matar, On viscous beads flowing down a vertical fibre, J. Fluid Mech. 553 (2006), pp. 85-105.

[3] L. B. Smolka, J. North, and B. K. Guerra, Dynamics of free surface perturbations along an annular viscous film, Phys. Rev. E 77 (2008), 036301.

[4] C. Duprat, C. Ruyer-Quil, S. Kalliadasis, and F. Giorgiutti-Dauphine, Absolute and convective instabilities of a viscous film flowing down a vertical fiber, Phys. Rev. Lett. 98 (2007), 244502.

[5] G. M. Sisoev, R. V. Craster, O. K. Matar, and S. V. Gerasimov, Film flow down a fibre at moderate flow rates, Chem. Eng. Sci. 61 (2006), pp. 7279-7298.

[6] C. Ruyer-Quil, P. Treveleyan, F. Giorgiutti-Dauphine, C. Duprat, and S. Kalliadasis, Modelling film flows down a fibre, J. Fluid Mech. 603 (2008), pp. 431-462.

[7] D. Quere, Fluid coating on a fiber, Annu. Rev. Fluid Mech. 31 (1999), pp. 347-384.

[8] A. DE RYCK and D. Quere, Fluid coating from a polymer solution, Langmuir 14 (1998), pp. 1911-1914.

[9] E. Ruckenstein, Scaling analysis of coating of a plate or a fiber, J. Colloid Interface Sci. 246 (2002), pp. 393-400.

[10] A. DE RYCK and D. QueRE, Inertial coating of a fiber, J. Fluid Mech. 311 (1996), pp. 219-237.

[11] J. B. Keller, S. I. Rubinow, and Y. O. Tu, Spatial instability of a jet, Phys. Fluids 16 (1973), pp. $2052-2055$.

[12] E. B. Dussan V, The moving contact line: the slip boundary-condition, J. Fluid Mech. 77 (1976), pp. 665-684.

[13] W. Cheney and D. Kincaid, Numerical Mathematics and Computing, Sixth Edition, Brooks Cole, New York, 2008.

[14] W. H. Press, S. A. Teukolsky, W. T. Vetterling, and B. P. Flannery, Numerical Recipes 3rd Edition: The Art of Scientific Computing, Cambridge University Press, New York, 2007.

[15] D. J. Acheson, Elementary Fluid Mechanics, Oxford University Press, Oxford, 1990.

[16] R. V. Craster and O. K. Matar, Dynamics and stability of thin liquid films, Rev. Mod. Phys. 81 (2009), pp. 1131-1198. 